\title{
STRONG MEASURE ZERO AND STRONGLY MEAGER SETS
}

\author{
TIMOTHY J. CARLSON
}

(Communicated by Andreas R. Blass)

\begin{abstract}
We consider conjectures made by Prikry and Galvin concerning strong measure zero and strongly meager sets of real numbers.
\end{abstract}

A set of real numbers $X$ is said to be of strong measure zero if for every sequence of positive numbers $\varepsilon_{0}, \varepsilon_{1}, \varepsilon_{2}, \ldots$ there are intervals $I_{0}, I_{1}, I_{2}, \ldots$ of corresponding lengths whose union contains $X$. Galvin, Mycielski, and Solovay proved a conjecture of Prikry by showing that a set is of strong measure zero iff it is contained in a translate of every comeager set. Prikry defined a set of real numbers to be strongly meager if it is contained in a translate of every set of full Lebesgue measure. He conjectured that the strongly meager sets form a $\sigma$-ideal and that it is consistent that the strongly meager sets are exactly the countable sets.

In the second section of this paper, the strong measure zero sets are shown to be closed under unions of size $\kappa$ under the assumptions of $\mathrm{MA}_{\kappa}$, verifying a conjecture of Prikry.

The notion of strong measure zero generalizes to arbitrary metric spaces in the obvious way: a metric space $X$ is of strong measure zero if for every sequence $\varepsilon_{0}, \varepsilon_{1}, \varepsilon_{2}, \ldots$ of positive numbers there are sets $X_{0}, X_{1}, X_{2}, \ldots$ whose union is $X$ such that the diameter of $X_{n}$ is at most $\varepsilon_{n}$ for all $n$. The Borel conjecture is that every strong measure zero set of reals is countable. Section 3 contains a proof that the Borel conjecture implies every strong measure zero metric space is countable. This had been conjectured by Galvin.

The statement that every strongly meager set of reals is countable is referred to as the dual Borel conjecture. Both the Borel conjecture and the dual Borel conjecture are consistently false. In fact, they both fail assuming the continuum hypothesis. Laver proved the consistency of the Borel conjecture; in $\S 4$ the consistency of the dual Borel conjecture is established.

An attempt to verify that the strongly meager sets form a $\sigma$-ideal led to the result that those sets of reals that are contained in a translate of every $G_{\delta}$ set of full measure form a $\sigma$-ideal. Section 5 contains a proof of this result.

Received by the editors March 20, 1989 and, in revised form, September 25, 1991. 1991 Mathematics Subject Classification. Primary 03E05, 03E15; Secondary 03E35, $03 E 50$.

Research supported by the NSF. 


\section{Preliminaries}

The notation used in this paper is mostly standard. For background on settheoretic notation consult [3].

The notions of strong measure zero and strongly meager are given above. Every nonseparable metric space has an uncountable collection of points with the distance between any two uniformly bounded away from 0 . So every strong measure zero metric space must be separable. Also, any subspace of a strong measure zero space is strong measure zero. Similarly, any subset of a strongly meager set is strongly meager.

We will often find it convenient to consider spaces other than the reals. $\mathbf{C}$ is the Cantor space, i.e., the collection of all characteristic functions of subsets of $\omega$ ( $\omega$ is the set of natural numbers). Define addition on $\mathbf{C}$ coordinatewise, where addition on the coordinates is $\bmod 2 . \mathbf{K}$ is $\mathbf{R} / \mathbf{Z}$, where $\mathbf{Z}$ is the set of integers and $\mathbf{R}$ is the set of reals. We can generalize the notion of strongly meager to any group with a translation invariant measure: if $G$ is such a group and $X$ is a subset of $G$, then $X$ is strongly meager iff $X$ is contained in a translate of each set of full measure. In this way we can speak of strongly meager subsets of $\mathbf{C}, \mathbf{R}$, and $\mathbf{K}$ (endowed with the usual Lebesgue measures).

\section{ADDITIVITY OF THE IDEAL OF STRONG MEASURE ZERO SETS}

Clearly, the strong measure zero sets are closed under countable unions. The following theorem verifies Prikry's conjecture that Martin's axiom implies that strong measure zero sets are closed under any union of size less than $2^{\aleph_{0}}$.

2.1. Theorem. Assuming $\mathbf{M A}_{\kappa}$, the strong measure zero sets are closed under unions of size $\kappa$.

Proof. Assume $\mathrm{MA}_{\kappa}$ and let $X_{\alpha}$ be of strong measure zero for $\alpha \in \kappa$. Let $X$ be the union of the $X_{\alpha}$. In order to show $X$ is of strong measure zero, suppose $\varepsilon_{0}, \varepsilon_{1}, \varepsilon_{2}, \ldots$ is a decreasing sequence of positive numbers. Define $\mathscr{P}$ to be the partial order consisting of all sets $p$ of open intervals with rational end points with the property that at most $n$ elements of $p$ have length greater than $\varepsilon_{n}$ for each $n$ and if $m_{n}$ is the number of elements of $p$ of length greater than $\varepsilon_{n}$ then the limit of $m_{n} / n$ is 0 . The ordering on $\mathscr{P}$ is reverse inclusion. Note that if $p$ is in $\mathscr{P}$ and the intervals in $p$ are $I_{0}, I_{1}, \ldots$, when listed so that the length of $I_{n+1}$ is no bigger than that of $I_{n}$ for each $n$, then the length of $I_{n}$ is at most $\varepsilon_{n}$.

Claim 1. $\mathscr{P}$ is c.c.c.

Suppose that $p_{\xi}$ is in $\mathscr{P}$ for $\xi \in \omega_{1}$. For each $\xi$ let $n_{\xi}$ have the property that if $m_{n, \xi}$ is the number of elements of $p_{\xi}$ of length greater than $\varepsilon_{n}$ then $m_{n, \xi} / n<1 / 2$ for all $n \geq n_{\xi}$. Let $p_{\xi}^{\prime}$ consist of the finitely many elements of $p_{\xi}$ of length greater than $\varepsilon_{n_{\xi}}$. Since there are only countably many choices for $n_{\xi}$ and $p_{\xi}^{\prime}$, there exist distinct $\xi$ and $\eta$ such that $n_{\xi}=n_{\eta}$ and $p_{\xi}^{\prime}=p_{\eta}^{\prime}$. $p_{\xi} \cup p_{\eta}$ is easily seen to be a condition in $\mathscr{P}$ that extends both $p_{\xi}$ and $p_{\eta}$. Therefore, $\mathscr{P}$ is c.c.c.

For $\alpha \in \kappa$ let $D_{\alpha}$ be the set of conditions $p$ in $\mathscr{P}$ such that $X_{\alpha}$ is contained in the union of the intervals in $p$.

Claim 2. $D_{\alpha}$ is dense. 
Let $p$ be a condition in $\mathscr{P}$. Let $m_{n}$ be the number of elements of $p$ of length greater than $\varepsilon_{n}$. Choose an increasing sequence $n_{i} \quad(i \in \omega)$ of density 0 such that for all $n, m_{n}+\left|\left\{i: n_{i}<n\right\}\right| \leq n$. Since $X_{\alpha}$ has strong measure zero, there is a sequence of open intervals with rational end points $I_{i} \quad(i \in \omega)$ such that $I_{i}$ has length at most $\varepsilon_{n_{i}}$ and $X_{\alpha}$ is contained in the union of the $I_{i}$.

Let $q$ be $p \cup\left\{I_{i}: i \in \omega\right\} . q$ is easily seen to be a condition and $X_{\alpha}$ is contained in the union of $q$.

Let $G$ be a filter on $\mathscr{P}$ that intersects each $D_{\alpha}$. Let $p^{*}$ be the union of $G$. Note that $p^{*}$ has at most $n$ elements of length greater than $\varepsilon_{n}$ for each $n$ since every element of $G$ has this property. Let $I_{0}, I_{1}, I_{2}, \ldots$ list the elements of $p^{*}$ such that the length of $I_{n+1}$ is no greater than that of $I_{n}$ for each $n$. As above, the length of $I_{n}$ is at most $\varepsilon_{n}$ for each $n$. Moreover, $X$ is contained in the union of the $I_{n}$ since each $X_{\alpha}$ is contained in the union of some element of $G$. Thus, $X$ is of strong measure zero.

The above proof is a modification, due to Galvin, of the original proof. The theorem generalizes to an arbitrary metric space, as shown in [2], by replacing open intervals with rational end points by elements of a countable base above. We have included the proof here for the convenience of the reader.

\section{MeTRIC SPACES AND Borel's CONJECTURE}

Galvin conjectured that Borel's conjecture implies every strong measure zero metric space is countable. This section verifies Galvin's conjecture.

3.1. Lemma. If $(X, d)$ is a separable metric space of cardinality less than $2^{\aleph_{0}}$ then there is a Lipschitz embedding of $(X, d)$ into the reals.

Proof. Let $x_{n} \quad(n \in \omega)$ enumerate a dense set in $(X, d)$. Define $d^{\prime}\left(x, x^{\prime}\right)$ to be the minimum of 1 and $d\left(x, x^{\prime}\right)$ for $x, x^{\prime} \in X$, and for each $x$ in $X$ let $h_{x}$ be the analytic function given by $\sum_{n \in \omega}\left(d^{\prime}\left(x, x_{n}\right) / n !\right) z^{n}$. If $x \neq x^{\prime}$ then there is an $n$ such that $d^{\prime}\left(x, x_{n}\right) \neq d^{\prime}\left(x^{\prime}, x_{n}\right)$, implying that $h_{x} \neq h_{x^{\prime}}$. Hence, if $x \neq x^{\prime}$ then $h_{x}$ and $h_{x^{\prime}}$ can agree on only countably many $z$. Since $X$ has cardinality less than $2^{\aleph_{0}}$, there is a positive real number $r$ such that $h_{x}(r) \neq h_{x^{\prime}}(r)$ for all distinct $x$ and $x^{\prime}$ in $X$. Define a map $h$ from $X$ into the reals by $h(x)=h_{x}(r)$.

Let $x$ and $x^{\prime}$ be elements of $X$.

$$
\begin{aligned}
\left|h(x)-h\left(x^{\prime}\right)\right| & \leq \sum_{n \in \omega}\left(\frac{\left|d^{\prime}\left(x, x_{n}\right)-d^{\prime}\left(x^{\prime}, x_{n}\right)\right|}{n !}\right) r^{n} \\
& \leq \sum_{n \in \omega}\left(\frac{d\left(x, x^{\prime}\right)}{n !}\right) r^{n}=d\left(x, x^{\prime}\right)\left[\sum_{n \in \omega}\left(\frac{1}{n !}\right) r^{n}\right] .
\end{aligned}
$$

Thus, $h$ has a Lipschitz constant of $e^{r}$.

3.2. Theorem. Assuming Borel's conjecture, every strong measure zero metric space is countable.

Proof. Suppose that $(X, d)$ is a metric space of strong measure zero. As remarked in $\S 1,(X, d)$ is separable. Arguing by contradiction, suppose that $X$ is uncountable. We may assume that $X$ has size $\aleph_{1}$. By Borel's conjecture, the continuum hypothesis fails. So by the previous lemma there is a Lipschitz embedding $h$ of $(X, d)$ into the real numbers. Let $c$ be a Lipschitz constant 
for $h$. For any subset $A$ of $X$, the diameter of $h(A)$ is at most $c d(A)$. This easily implies that $h(X)$ is of strong measure zero, contradicting Borel's conjecture.

The original proof of this theorem used Müntz's theorem. The proof here is a simplication by Galvin.

\section{Consistency of THE duAl Borel CONJeCture}

Since Laver showed the consistency of Borel's conjecture, Prikry's conjecture that the dual Borel conjecture is consistent seems natural. The consistency of the dual Borel conjecture is established in this section by adding Cohen reals to a model of ZFC. That this approach works is somewhat of a surprise since the duality of measure and category would suggest that Borel's conjecture could be obtained by adding random reals. But adding random reals to a model in which Borel's conjecture fails, one gets a generic extension in which Borel's conjecture still fails (however, in unpublished notes Woodin has shown that Borel's conjecture can be preserved by adding random reals).

Notice that the dual Borel conjecture follows if every strongly meager subset of $\mathbf{K}$ is countable since the residues mod $\mathbf{Z}$ of any strongly meager subset of $\mathbf{R}$ is a strongly meager subset of $\mathbf{K}$.

We will need the following theorem of Lorentz [5].

4.1. Theorem. There exists a constant $C$ such that if $A \subseteq \mathbf{Z} / n \mathbf{Z}$ and $|A| \geq 2$ then there exists $B \subseteq \mathbf{Z} / n \mathbf{Z}$ with $|B| \leq C \cdot n \cdot \log |A| /|A|$ such that $A+B=\mathbf{Z} / n \mathbf{Z}$.

4.2. Corollary. If $X$ is a finite subset of $\mathbf{K}$ that has cardinality at least 2 then there is an open subset $U$ of $\mathbf{K}$ such that $X+U=\mathbf{K}$ and the measure of $U$ is at most $2 \cdot C \cdot \log |X| /|X|$.

Proof. Choose $m$ so large that any two elements of $X$ have distance greater than $1 / m$. Let $A$ be the collection of all integers $i$ in $[0, m)$ such that $X$ intersects $[i / m,(i+1) / m)$. Note that $|X|=|A|$. Let $B$ be the (representatives in $\{0,1, \ldots, m-1\}$ of the elements of the) set described in the conclusion of Theorem 4.1, and let $U$ be the union of the intervals $((j-1) / m,(j+1) / m)$ with $j \in B$.

4.3. Corollary. Suppose $X$ is a finite subset of $\mathbf{K}$ of cardinality at least 2 . There is a subset $P$ of $\mathbf{K}$ that is a finite union of closed intervals with rational end points such that $X$ is not contained in a translate of $P$ and the measure of $P$ is at least $1-2 \cdot C \cdot \log |X| /|X|$.

Proof. Let $U$ be as in the conclusion of Corollary 4.2. We may assume that $U$ is a finite union of open intervals with rational end points. Let $P$ be the complement of $-U$. The following claim implies $P$ is as required.

Claim. If $G$ is a group and $A$ and $B$ are subsets of $G$ such that $A+B=G$, then every translate of $A$ intersects $-B$.

Let $t \in G$. We want to see that $A+t$ meets $-B$. Choose $a \in A$ and $b \in B$ with $a+b=-t . a+t$ is in both $A+t$ and $-B$.

4.4. Definition. For a real $c$ in $[0,1), \mathscr{P}_{c}$ is the partial order consisting of all $P \subseteq \mathbf{K}$ of measure greater than $c$ such that $P$ is a finite union of closed intervals with rational end points. $\mathscr{P}_{c}$ is ordered by inclusion. 
Note that forcing with $\mathscr{P}_{c}$ is equivalent to adding a Cohen real.

4.5. Definition. Suppose that $G$ is a $V$-generic filter on $\mathscr{P}_{c}$. The compact set determined by $G$ is the intersection of $G$ as calculated in $V[G]$.

A word of explanation is required for the previous definition. Each element $P$ of $\mathscr{P}_{c}$ is of the form $\left[a_{0}, b_{0}\right] \cup\left[a_{1}, b_{1}\right] \cup \cdots \cup\left[a_{k}, b_{k}\right]$ (calculated in $V$ ) for some rational numbers $a_{0}, b_{0}, a_{1}, b_{1}, \ldots, a_{k}, b_{k}$. The compact set determined by $G$ is the intersection of all the sets $P^{V[G]}$, where $P \in G$ and $P^{V[G]}$ is the set $\left[a_{0}, b_{0}\right] \cup\left[a_{1}, b_{1}\right] \cup \cdots \cup\left[a_{k}, b_{k}\right]$ as calculated in $V[G]$ (using the notation above). Note that this set has measure $c$.

4.6. Lemma. Let $G$ be a $V$-generic filter on $\mathscr{P}_{c}$ and let $K$ be the compact set of measure $c$ determined by $G$. In $V[G]$ no translate of $K$ has uncountable intersection with $\mathbf{K}^{V}$.

Proof. Working in $V[G]$, suppose $X \subseteq \mathbf{K}^{V}$ is uncountable and contained in a translate of $K$. Since $\mathscr{P}_{c}$ is countable, there is an uncountable subset of $X$ that is an element of $V$. So we may assume that $X \in V$. Let $P$ be a condition in $G$ that forces " $X$ is contained in a translate of $\underline{K}$ " where $\underline{K}$ is the canonical name for the compact set added by $G$, i.e., every condition forces " $\underline{K}$ is the compact set determined by $\underline{G}$ " where $\underline{G}$ is the canonical name for the generic filter. Choose $n$ so that $2 \cdot C \cdot \log (n) / n<p-c$, where $p$ is the measure of $P$. Let $X^{*}$ be a finite subset of $X$ of size $n$. By the previous lemma, there is an element $P_{0}$ of $\mathscr{P}_{c}$ of measure at least $1-2 \cdot C \cdot \log (n) / n$, no translate of which contains $X^{*}$. Let $P_{1}$ be $P \cap P_{0} . P_{1}$ is an element of $\mathscr{P}_{c}$. By absoluteness, every condition forces that $X^{*}$ is not contained in a translate of $P_{1}$. Therefore, $P_{1}$ forces " $X^{*}$ is not contained in a translate of $\underline{K}$," which is a contradiction since $P_{1}$ is stronger than $P$.

4.7. Theorem. If $\mathscr{C}$ is the partial order for adding $\kappa$ Cohen reals, where $\kappa$ is at least $\aleph_{2}$, then the dual Borel conjecture is true in $V^{\mathscr{C}}$.

Proof. Suppose that $G$ is a $V$-generic filter on $\mathscr{C}$. In order to reach a contradiction, assume that the dual Borel conjecture fails in $V[G]$. By remarks above, there is a subset $X$ of $\mathbf{K}$ of size $\aleph_{1}$ in $V[G]$ that is strongly meager. Let $r_{\xi}$ be the $\xi$ th Cohen real added by $G$. There is a proper subset $J$ of $\kappa$ such that $X$ is an element of $V^{\prime}=V\left[\left\langle r_{\xi}: \xi \in J\right\rangle\right]$ (of course, there are such $J$ of size $\aleph_{1}$ ). We may assume that $\kappa \backslash J$ has exactly one element so that $V[G]$ is an extension of $V^{\prime}$ by the addition of one Cohen real. Since $X$ is a subset of $\mathbf{K}^{V^{\prime}}$, Lemma 4.6 implies that for any rational $c \in[0,1), V[G] \vDash$ "there is a subset of $\mathbf{K}$ of measure $c$ no translate of which has uncountable intersection with $X$." This clearly implies that $V[G] \vDash$ "there is a subset of $\mathbf{K}$ of measure 1 no translate of which has uncountable intersection with $X$." Therefore, $V[G] \vDash$ " $X$ is not strongly meager," which is a contradiction.

\section{ARE THE STRONGLY MEAGER SETS CLOSED UNDER FINITE UNIONS?}

This question, raised by Prikry after defining the notion of strongly meager set, is open. Theorem 5.11 says that no perfect set can be the union of countably many strongly meager sets. 
5.1. Problem. If $A$ is a subset of $\mathbf{K}$ of full measure then there is a subset $B$ of $\mathbf{K}$ of full measure such that any countable union of translates of $B$ is contained in a translate of $A$.

A positive solution to this problem implies that the union of countably many strongly meager sets is strongly meager. More generally, if "countable" is replaced by " $\kappa$ many" we can conclude that the union of $\kappa$ strongly meager sets is strongly meager. For suppose that $X_{i}$ is strongly meager for $i \in \kappa$ and let $X$ be the union of the $X_{i}$. Now suppose that $A$ is a set of full measure and get $B$ from Problem 5.1. For each $i \in \kappa$ there is a translate of $B$ that contains $X_{i}$. These translates of $B$ are contained in a single translate of $A$ that must also contain $X$. Therefore, $X$ is strongly meager.

5.2. Definition. Suppose $\kappa$ is a cardinal number and $\mathscr{U}$ is a set of subsets of a group $\mathbf{G} . \mathscr{U}$ is $\kappa$-translatable if for every $A$ in $\mathscr{U}$ there is a $B$ in $\mathscr{U}$ such that the union of any $\kappa$ many translates of $B$ is contained in a translate of $A$.

Using this definition, Problem 5.1 asks whether the filter of full measure sets is $\aleph_{0}$-translatable. Notice that if $\mathscr{U}$ is a filter then $\mathscr{U}$ is $\kappa$-translatable iff for every $A$ in the dual ideal there is a $B$ in the dual ideal such that the intersection of any $\kappa$ translates of $B$ contains a translate of $A$. Hence, $\mathscr{U}$ is $\kappa$-translatable iff for every $A$ in the dual ideal there is $B$ in the dual ideal such that the union of any $\kappa$ many translates of $A$ is contained in a translate of $B$.

5.3. Definition. Suppose $\kappa$ is a cardinal number. A subset $X$ of a group $\mathbf{G}$ is $\kappa$-covering if every subset $Y$ of $\mathbf{G}$ of size $\kappa$ is contained in a translate of $X$.

5.4. Lemma. Suppose $\mathscr{U}$ is a filter on a group $\mathbf{G} . \mathscr{U}$ is $\kappa$-translatable iff for every set $A$ in the dual ideal of $\mathscr{U}$ there is a $\kappa$-covering set $X$ such that $A+X$ is in the dual ideal of $\mathscr{U}$.

Proof. Suppose $A$ and $B$ are subsets of $\mathbf{G}$ and define $X$ to consist of all $x$ such that $A+x \subseteq B$. Notice that $A+y \subseteq B-t$ for all $y \in Y$ iff $Y+t \subseteq X$. Hence, $X$ is $\kappa$-covering iff

The union of any $\kappa$ many translates of $A$ is contained in a translate of $B$

As remarked earlier, $\mathscr{U}$ is $\kappa$-translatable iff for every $A$ in the dual ideal there is a $B$ in the dual ideal such that $(*)$ holds. The lemma follows easily.

By the lemma, Problem 5.1 is equivalent to

5.5. Problem. If $A$ is a subset of $\mathbf{K}$ of measure 0 then there is an $\aleph_{0}$-covering set $X$ such that $A+X$ has measure 0 .

We will see that the dual of Problem 5.1 is true, i.e., the filter of comeager sets is $\aleph_{0}$-translatable; first we will prove that the filter generated by the $G_{\delta}$ sets of full measure is $\aleph_{0}$-translatable.

5.6. Lemma. Let $\mathbf{G}$ be either $\mathbf{K}$ or $\mathbf{C}$. If $A$ is an $F_{\sigma}$ subset of $\mathbf{G}$ of measure 0 then there is an $\aleph_{0}$-covering $F_{\sigma}$ set $X$ such that $A+X$ has measure 0. 
Proof. We will first give the proof for $A$ which is a subset of $\mathbf{C}$, since it is clearer, and then describe the modifications required for $\mathbf{K}$.

For $I$, a subset of $\omega$, let $X[I]$ be the closed set consisting of all $x$ in $\mathbf{C}$ such that $x(i)=0$ for all $i$ in $I$.

Claim 1. If $I_{n} \quad(n \in \omega)$ is a collection of pairwise disjoint subsets of $\omega$ and $X$ is the union of the sets $X\left[I_{n}\right] \quad(n \in \omega)$, then $X$ is $\aleph_{0}$-covering.

To prove the claim, suppose $x_{n}(n \in \omega)$ is a countable collection of elements of $\mathbf{C}$. Let $t$ be an element of $\mathbf{C}$ such that $t \uparrow I_{n}=x_{n} \uparrow I_{n}$. Then $t+x_{n}$ is 0 on $I_{n}$, i.e., $t+x_{n} \in X\left[I_{n}\right]$. So $\left\{x_{n}: n \in \omega\right\}+t \subseteq X$.

Claim 2. If $B$ is a closed set of measure 0 and $a \in \omega$, then there exists $b \in \omega$ such that $B+X[(a, b)]$ has arbitrarily small measure.

The claim is straightforward, noting that, since $X[[a, \omega)]$ is finite, $B+$ $X[[a, \omega)]$ has measure zero and is the intersection of the sets $B+X[[a, b)]$.

To prove the theorem for $\mathrm{C}$, suppose $A$ is the union of closed sets $B_{n}$ $(n \in \omega)$ that have measure zero. Using Claim 2, construct pairwise disjoint sets $I_{n}$ such that $B_{n}+X\left[I_{m}\right]$ has measure zero for all $n$ and $m$. Let $X$ be the union of the $X\left[I_{n}\right]$.

To verify the theorem for $\mathbf{K}$, we modify the definition of $X[I]$ using binary expansions. For $n \neq 0$ let $F_{n}$ be the union of the intervals $\left[i / 2^{n},(i+1) / 2^{n}\right]$, where $i$ is even and $i<2^{n}$. We now let $X[I]$ be $\bigcap\left\{F_{n}: n \in I\right\}$.

Claim 3. Suppose $H$ is a closed interval of length $2^{-n}, x \in \mathbf{K}$, and $n<k$. There is a closed subinterval $H^{*}$ of $H$ of length $2^{-k-1}$ such that $H^{*}+x \subseteq$ $X[(n, k]]$.

To prove the claim, note that $X[(n, k]]=\bigcap\left\{F_{j}: n<j \leq k\right\}$ is the union of the intervals of the form $\left[i / 2^{n}, i / 2^{n}+1 / 2^{k}\right] . H+x$ is long enough that it must overlap at least one of these intervals for half its length. Now choose $H^{*}$ so that $H^{*}+x$ will be contained in this overlap.

Claim 4. Let $k_{i} \quad(i \in \omega)$ be a strictly increasing sequence of natural numbers with $k_{0}=0$. Suppose $I_{n}(n \in \omega)$ is a collection of pairwise disjoint subsets of $\omega$, each of which is a union of intervals of the form $\left(k_{i}, k_{i+1}\right)$. If $X$ is the union of the sets $X\left[I_{n}\right] \quad(n \in \omega)$ then $X$ is $\aleph_{0}$-covering.

Suppose $x_{n} \in \mathbf{K}$ for $n \in \omega$. Inductively choose a decreasing sequence of closed intervals $H_{i}$ of length $2^{-k_{i}}$ such that if $\left(k_{i}, k_{i+1}\right)$ is contained in $I_{n}$ then $H_{i}+x_{n} \in X\left[\left(k_{i}, k_{i+1}\right)\right]$. This is possible by Claim 3. If $t$ is in the intersection of the $H_{i}$ then $x_{n}+t \in X\left[I_{n}\right] \subseteq X$ for all $n$. This proves the claim.

Claim 2 remains valid in this context and the rest of the argument goes through as before.

5.7. Theorem. Let $\mathbf{G}$ be either $\mathbf{K}$ or $\mathbf{C}$. If $A$ is a $G_{\delta}$ subset of $\mathbf{G}$ of full measure then there is $a G_{\delta}$ set $B$ of full measure such that the union of any countably many translates of $B$ is contained in a translate of $A$.

Proof. Immediate from Lemmas 5.4 and 5.6.

5.8. Corollary. If $A$ is $a G_{\delta}$ subset of $\mathbf{R}$ of full measure then there is a $G_{\delta}$ subset $B$ of $\mathbf{R}$ of full measure such that the union of any countably many translates of $B$ is contained in a translate of $A$. 
Proof. Straightforward, since there is no loss of generality in assuming that $A$ has period 1.

5.9. Corollary. Let $\mathbf{G}$ be either $\mathbf{K}, \mathbf{R}$, or $\mathbf{C}$. The collection of subsets of $\mathbf{G}$ that are contained in a translate of every $G_{\delta}$ of full measure form a $\sigma$-ideal.

Proof. Using the theorem and corollary, this follows by the same argument given above showing the strongly meager sets are a $\sigma$-ideal provided Problem 5.1 is true.

Using the following result of Erdös, Kunen, and Mauldin from [1] we will be able to conclude that no perfect set is the union of countably many strongly meager sets.

5.10. Theorem. Let $\mathbf{G}$ be either $\mathbf{K}$ or $\mathbf{R}$. If $A$ is a perfect subset of $\mathbf{G}$ there is a closed set of measure zero $B$ such that $A+B=\mathbf{G}$.

The corresponding result for $\mathbf{C}$ also holds by essentially the same proof (while some technical complications are avoided). We sketch the argument here. We will need the fact that Lorentz's proof of Theorem 4.1 works for arbitrary groups rather than just groups of the form $\mathbf{Z} / n \mathbf{Z}$; i.e., there is a constant $C$ such that for any finite group $G$ and any subset $A$ of $G$ with at least two elements there is a subset $B$ of $G$ of size at most $C \cdot|G| \cdot \log |A| /|A|$ such that $A+B=G$.

Suppose $A$ is a perfect subset of $\mathbf{C}$. Let $T$ be the tree of finite sequences corresponding to $A$, i.e., $T$ consists of all finite sequences from $\{0,1\}$ that extend to an element of $A$. Choose a sequence $n_{i}(i \in \omega)$ that grows fast enough that whenever $s$ is a sequence of length $n_{i}$ in $T$ then the number of extensions of $s$ of length $n_{i+1}$ in $T$ is large. Let $G_{i}$ be the finite group $\{0,1\}^{\left[n_{i}, n_{i+i}\right)}$ with coordinatewise operations. We will choose a subset $S_{i}$ of $G_{i}$ for each $i$ and then let $B$ consists of all $x$ such that the restriction of $x$ to $\left[n_{i}, n_{i+1}\right)$ is in $S_{i}$ for all $i$. Fix $i$, and for each $s$ in $T$ of length $n_{i}$ let $A_{s}$ be the collection of $t \uparrow\left[n_{i}, n_{i+1}\right)$, where $t$ is an element of $T$ of length $n_{i+1}$ that extends $s$. For each such $s$ there is a small set $B_{s}$ such that $A_{s}+B_{s}=G_{i}$. Let $S_{i}$ be the union of the $B_{s}$. $S_{i}$ will be small, being a small union of small sets. Hence, $B$ will have measure zero. A little thought shows that $A+B=\mathrm{C}$.

5.11. Theorem. Let $\mathbf{G}$ be either $\mathbf{K}, \mathbf{R}$, or $\mathbf{C}$. No perfect subset of $\mathbf{G}$ is the union of countably many strongly meager sets.

Proof. Suppose $A$ is a perfect subset of $\mathbf{G}$. By Theorem 5.10 and its analogue for $\mathbf{C}$, there is a closed set $B$ of measure 0 such that $A+B=\mathbf{G}$. Let $U$ be the complement of $-B . U$ is an open set of full measure that, by the claim in the proof of Corollary 4.3, does not contain a translate of $A$. So $A$ is not in the $\sigma$-ideal described in Corollary 5.9. Since every strongly meager set is in this ideal, no perfect set is the union of countably many strongly meager sets.

The techniques used above to show that the filter generated by the full measure $G_{\delta}$ sets in $\mathbf{K}, \mathbf{R}$, or $\mathbf{C}$ is $\aleph_{0}$-translatable can be used to show that the filter of comeager sets is $\aleph_{0}$-translatable. For $\mathbf{K}$ and $\mathbf{R}$, this also follows from the theorem below, which is implicit in the work of Galvin, Mycielski, and Solovay (see [6]). 
5.12. Theorem. If $A$ is a comeager subset of $\mathbf{K}$ there are positive real numbers $\varepsilon_{0}, \varepsilon_{1}, \varepsilon_{2}, \ldots$ such that whenever $I_{n}$ is an open interval of length at most $\varepsilon_{n}$ for all $n$ then lim $\sup _{n \in \omega} I_{n}$ is contained in a translate of $A$.

Proof. Let $D_{n} \quad(n \in \omega)$ be a decreasing collection of dense open sets whose intersection is contained in $A$. Define $\varepsilon_{n}$ by induction on $n$ such that whenever $I_{0}, I_{1}, \ldots, I_{n}$ is a sequence of closed intervals such that $I_{n}$ has length at most $\varepsilon_{n}$ then there is a real $t$ such that $I_{i}$ is contained in $D_{i}+t$ for $i=0, \ldots, n$.

Suppose that $\varepsilon_{i}$ has been chosen for $i<n$. For $\left(x_{0}, \ldots, x_{n-1}\right) \in \mathbf{K}^{n}$ the set $\left\{t:\left[x_{i}, x_{i}+\varepsilon_{i}\right] \subseteq D_{i}+t\right.$ for $\left.i<n\right\}$ is open and nonempty. Since $D_{i}$ is dense and open, $\left\{t:\left[x_{i}, x_{i}+\varepsilon_{i}\right] \subseteq D_{i}+t\right.$ for $i<n$ and $\left.x_{n} \in D_{n}+t\right\}$ is nonempty. Therefore, if $U_{t}$ is defined to be the set of all $\left(x_{0}, \ldots, x_{n}\right) \in \mathbf{K}^{n+1}$ such that $\left[x_{i}, x_{i}+\varepsilon_{i}\right] \subseteq D_{i}+t$ for $i<n$ and $x_{n} \in D_{n}+t$, then $U_{t}(t \in \mathbf{K})$ is an open cover of $\mathbf{K}$. The Lebesgue covering lemma implies that an appropriate $\varepsilon_{n}$ exists.

To see that the sequence of $\varepsilon_{n}$ is as required, let $I_{n}$ be an open interval of length at most $\varepsilon_{n}$ for $n \in \omega$. Let $F_{n}$ be the collection of those $t$ such that $I_{n} \subseteq D_{n}+t$. Since $I_{n}$ is open, $F_{n}$ is closed. Moreover, the collection of $F_{n}$ has the finite intersection property by the choice of the $\varepsilon_{n}$. Choose $t$ in the intersection of all $F_{n}$. So $I_{n} \subseteq D_{n}+t$ for all $n$. If $x$ is in infinitely many $I_{n}$ then $x-t \in D_{n}$ for infinitely many $n$ implying $x-t \in A$. Therefore, $\lim \sup _{n \in \omega} I_{n} \subseteq A+t$.

5.13. Theorem. If $A$ is a comeager subset of $\mathbf{K}$ there is a comeager subset $B$ of $\mathbf{K}$ such that the union of any countably many translates of $B$ is contained in a translate of $A$.

Proof. Suppose that $A$ is a comeager subset of $\mathbf{K}$. Let $\varepsilon_{n} \quad(n \in \omega)$ be as in the previous theorem. Let $J_{k}(k \in \omega)$ be infinite pairwise disjoint subsets of $\omega$. Choose open intervals $I_{n}(n \in \omega)$ such that $I_{n}$ has length at most $\varepsilon_{n}$ and $\bigcup\left\{I_{m}: n \leq m\right.$ and $\left.m \in J_{k}\right\}$ is dense for each $n$ and $k$. Now define $B_{k}$ to be limsup $\operatorname{suJ}_{n} I_{n}$ and let $B$ be the intersection of the $B_{k}$. Each $B_{k}$ is a dense $G_{\delta}$ so $B$ is also a dense $G_{\delta}$.

To see that $B$ satisfies the conclusion of the theorem, let $t_{k} \in \mathbf{K}$ for $k \in \omega$. The theorem requires there be a translation of $A$ that contains $B+t_{k}$ for each $k$. For this, it suffices to find a translate of $A$ that contains $B_{k}+t_{k}$ for each $k$. Define $I_{n}^{*}$ to be $I_{n}+t_{k}$ if $n \in J_{k}$. There is a $t \in \mathbf{K}$ such that $\lim \sup _{n \in \omega} I_{n}^{*} \subseteq$ $A+t$. In particular, $B_{k}+t_{k}=\left(\lim \sup _{n \in J_{k}} I_{n}\right)+t_{k}=\lim \sup _{n \in J_{k}} I_{n}^{*} \subseteq A+t$ for each $k$.

5.14. Corollary. If $A$ is a comeager subset of $\mathbf{R}$ then there is a comeager subset $B$ of $\mathbf{R}$ such that the union of any countably many translates of $B$ is contained in a translate of $A$.

Proof. Straightforward, since there is no loss of generality in assuming that $A$ has period 1 .

\section{REFERENCES}

1. P. Erdős, K. Kunen, and R. D. Mauldin, Some additive properties of sets of real numbers, Fund. Math. 113 (1981), 187-199.

2. D. H. Fremlin, Consequences of Martin's axiom, Cambridge Univ. Press, London and New York, 1984. 
3. T. Jech, Set theory, Academic Press, New York, 1978.

4. R. Laver, On the consistency of Borel's conjecture, Acta Math. 137 (1976), 151-169.

5. G. G. Lorentz, On a problem of additive number theory, Proc. Amer. Math. Soc. 5 (1954), 838-841.

6. A. Miller, Special subsets of the real line, Handbook of Set-Theoretic Topology (K. Kunen and J. E. Vaughan, eds.), North-Holland, Amsterdam, 1984.

Department of Mathematics, Ohio State University, Columbus, Ohio 43210-1328

E-mail address: carlson@function.mps.ohio-state.edu 